\title{
ELEMENTAL AND ISOTOPIC ANALYSIS OF URANIUM OXIDE AND NIST GLASS STANDARDS \\ BY FEMTOSECOND-LA-ICP-MIC-MS
}

\author{
Ames Laboratory-USDOE \\ Report IS-5194
}

Chris Ebert, Daniel S. Zamzow, Eddie H. McBay, ${ }^{\#}$ Debra A. Bostick, ${ }^{\#}$ Stanley J. Bajic, David P. Baldwin, and R. S. Houk

Ames Laboratory-USDOE

Department of Chemistry

Iowa State University

Ames, IA 50011
\# Oak Ridge National Laboratory-USDOE Oak Ridge, TN 37831

June 2009 


\section{Introduction}

The objective of this work was to test and demonstrate the analytical figures of merit of a femtosecond-laser ablation ( $f s-\mathrm{LA}$ ) system coupled with an inductively coupled plasma-multiion collector-mass spectrometer (ICP-MIC-MS). The mobile fs-LA sampling system was designed and assembled at Ames Laboratory and shipped to Oak Ridge National Laboratory (ORNL), where it was integrated with an ICP-MIC-MS. The test period of the integrated systems was February 2-6, 2009.

Spatially-resolved analysis of particulate samples is accomplished by 100-shot laser ablation using a fs-pulsewidth laser and monitoring selected isotopes in the resulting ICP-MS transient signal. The capability of performing high sensitivity, spatially resolved, isotopic analyses with high accuracy and precision and with virtually no sample preparation makes fsLA-ICP-MIC-MS valuable for the measurement of actinide isotopes at low concentrations in very small samples for nonproliferation purposes. Femtosecond-LA has been shown to generate particles from the sample that are more representative of the bulk composition, thereby minimizing weaknesses encountered in previous work using nanosecond-LA (ns-LA). ${ }^{1-3}$ The improvement of $f s$ - over $n s$-LA sampling arises from the different mechanisms for transfer of energy into the sample in these two laser pulse-length regimes. The shorter duration $f s$-LA pulses induce less heating and cause less damage to the sample than the longer ns pulses. This results in better stoichiometric sampling (i.e., a closer correlation between the composition of the ablated particles and that of the original solid sample), which improves accuracy for both intraand inter-elemental analysis.

The primary samples analyzed in this work are a) solid uranium oxide powdered samples having different ${ }^{235} \mathrm{U}$ to ${ }^{238} \mathrm{U}$ concentration ratios, and b) glass reference materials (NIST 610, 
612, 614, and 616). Solid uranium oxide samples containing ${ }^{235} \mathrm{U}$ in depleted, natural, and enriched abundances were analyzed as particle aggregates immobilized in a collodion substrate. The uranium oxide samples were nuclear reference materials (CRMs U0002, U005-A, 129-A, U015, U030-A, and U050) obtained from New Brunswick Laboratory-USDOE.

\section{Experimental}

Femtosecond-LA sampling was accomplished using a Coherent Libra fs-laser system with UV harmonic generation. The Libra laser system was placed on a $1.83-\mathrm{m}$ x $0.91-\mathrm{m}$ steel cart along with its ancillary components (e.g., power supplies and water recirculator). The 266nm output from the Libra was directed through a beam tube into an interlocked enclosure, which was also located on the cart. The enclosure housed optics, an electronic shutter, gas lines, and valves for the argon carrier gas, an $x-y-z$ translation stage, and the ablation cell. Since the entire laser beam path was enclosed in beam tubes and an interlocked enclosure, laser ablation sampling was performed in a Class-I laser hazard mode. Class-I signifies that the LA system is considered safe from any potential laser exposure hazards to operators. Having the fs-LA system on a cart allowed the system to be moved into a separate room where it could be operated in a non-Class-I mode safely during initial set-up and optimization at ORNL.

The Ti:Sapphire output from the Libra laser was frequency-tripled to a wavelength of 266 $\mathrm{nm}$, with an energy of $180 \mu \mathrm{J}$ per pulse, a pulse width of approximately $100 \mathrm{fs}$, and a repetition rate of $1 \mathrm{kHz}$. The laser beam spot size used in these tests was approximately $35 \mu \mathrm{m}$. A programmable shutter was used to control the number of laser pulses for a particular ablation event. A 100-ms shutter-open duration was used (i.e., 100 laser shots) for the uranium oxide measurements. The shutter-open duration was varied from 10 -ms to 500 -ms for the single-spot 
analyses of the glass reference samples. Only results using a 100-ms shutter-open duration are reported here.

The ICP-MS instrument used at ORNL was a Thermo Finnigan NEPTUNE ICP-MS equipped with a multi-ion collector (MIC) detector array and a Faraday cup detector array. The MIC array configuration used during the test period is shown in Table I. Table II shows the Faraday cup configuration used. Thorium-232 was added to the Faraday cup array during some of the testing to detect Th for the inter-element measurement of the glass reference samples. The detector integration time was 131-ms for all transient measurements, generally using 400 points ( 52 s) for each LA run. The ICP-MIC-MS was operated in low resolution mode for maximum sensitivity. Other instrumental laser ablation and ICP-MS parameters were optimized daily. ICP-MS operating conditions were chosen to maximize $\mathrm{U}^{+}$signal from a nebulized aqueous solution of $\sim 100 \mathrm{ppb} \mathrm{U}$.

Samples for laser ablation sampling were prepared by spreading very small amounts of uranium oxide powder onto an acrylic disk, which had a 2-cm diameter with a 2-mm deep depression on one face. The powder was suspended in a less than $200-\mu \mathrm{m}$ thick layer of collodion, at a loading of approximately 5-mg of uranium oxide per disk. Single-spot analyses of the samples were restricted to an approximate 35- $\mu \mathrm{m}$ diameter area for each run, the diameter of the focused laser beam. This was the beam diameter selected for these tests, but not the minimum size for this instrument.

\section{Results and Discussion}

Uranium Isotope Ratios. Figure 1 shows representative transient signals acquired from a 100shot $f$-LA-ICP-MIC-MS analysis of a 1.5\% enriched uranium oxide sample (U015). The ${ }^{234} \mathrm{U}$, ${ }^{235} \mathrm{U},{ }^{236} \mathrm{U}$, and ${ }^{238} \mathrm{U}$ signal intensities are plotted on a logarithmic scale for clear visualization. 
(The observed gaps in the plotted traces at low signal intensities are due to occasional negative values, which cannot be plotted on a logarithmic scale.) From these traces, the ${ }^{235} \mathrm{U} /{ }^{238} \mathrm{U}$ ratios were determined for the set of depleted, natural, and enriched uranium oxide samples. Since pure uranium oxide samples were analyzed to establish the analytical figures of merit for the state-of-the-art fs-LA-ICP-MIC-MS system, strong ${ }^{238} \mathrm{U}$ signal intensities (>10 volts) were commonly observed during these tests. The strong ${ }^{238} \mathrm{U}$ signals required measurement of these samples using Faraday cups rather than the more sensitive electronmultiplier available on this instrument. The ion collectors have an upper voltage threshold of $\sim 3 \mathrm{mV}$ and would be readily saturated and the data useless for such larger ion concentrations. No measurable uranium background was detected for the collodion or acrylic substrate.

The results from the 100-shot fs-LA-ICP-MIC-MS for two days of analyses of the uranium oxide samples are shown in Table III. There is excellent agreement between the measured 100-shot laser ablation values and the certified values of the CRMs. The measured ratios include small corrections for mass bias; details of the method for such corrections can be obtained from the authors. The relative standard deviation (RSD) for the two data sets is less than $0.75 \%$ for multiple runs done for each of the uranium oxide samples. These results demonstrate the significant improvement in precision and accuracy that this state-of-the-art fsLA-ICP-MIC-MS provides over a more conventional commercial ns-LA-ICP-MS system. ${ }^{4}$

Table IV shows results for the analysis of the same uranium oxide samples using both a commercial $n s$ laser for ablation sampling (CETAC LSX-500) and the fs-LA system used during the site test, coupled with a sequential-scanning ICP-MS (Thermo Finnigan Element I) at Ames Laboratory. Please note that this is not data generated using the ICP-MIC-MS at ORNL. Even though the $f s$-LA system generally provides better results than the $n s$-LA system, poorer 
accuracy and precision are observed for either LA system when integrated to a sequentialscanning ICP-MS compared to the results obtained with the fs-LA-ICP-MIC-MS setup used during the site test.

A least-squares fit of the entire dataset was performed and the results are shown in Figure 2. (Figure 3 shows an expanded view of the fit around natural isotope abundance uranium.) The measured 100-shot fs-LA-ICP-MIC-MS data for the uranium oxide samples are plotted against their known values. The 95\% prediction and confidence intervals and the literature value for ${ }^{235} \mathrm{U} /{ }^{238} \mathrm{U}$ in natural uranium, $0.725 \%$, are also plotted. The linear-regression fit to the data yielded a correlation coefficient of $\mathrm{R}=0.99999$. From the data, one can determine that a single ablation measurement that yielded a value of $0.725 \%{ }^{235} \mathrm{U} /{ }^{238} \mathrm{U}$ (natural abundance) would be $95 \%$ likely to lie in the prediction range of 0.707 to $0.742 \%$. The $95 \%$ prediction interval from the previous study for the same hypothetical natural abundance sample, performed with a conventional commercial $n s$-LA unit and sequential ICP-MS, was 0.611 to $0.826 \%{ }^{4}$ This demonstrates a distinct improvement in precision (over six times more precise), due to the incorporation of $f s$-laser sampling and the multi-ion collector MS, thus meeting one of the project's most significant goals. The much higher precision obtained with the tested state-of-theart system means that error contributions from the plasma or laser are now the most significant limitation to improved accuracy, sensitivity, and precision. These error contributions may addressed by more frequent renormalization of the measured system response to isotopic standards. For example, the analytical protocol would likely be modified to perform normalization between each sample or after every third sample, rather than once per sample set as was done for the site test data, by performing a mass-bias correction. (Additional normalization data were not collected during the site test because of time limitations.) 
The least-squares fit in Figure 2 shows that a known natural abundance sample would yield a measurement within a 0.723 to $0.728 \%$ confidence range $95 \%$ of the time, determined from this calibration dataset. The same hypothetical natural abundance sample yielded a measurement within a 0.718 to $0.745 \%$ confidence range $95 \%$ of the time, in the previous study with poorer precision. ${ }^{4}$ The confidence intervals are significantly improved with the fs-LA-ICPMIC-MS system (0.005\% versus $0.027 \%)$. Thus, much smaller enrichment or depletion levels can be accurately measured using the fs-LA-ICP-MIC-MS system.

Thorium/Uranium Concentration Ratios. Measurement of the Th/U signal ratio is a convenient way to determine if the particles produced by the LA process are small enough to be effectively converted into atoms and ions in the ICP. A measured Th/U ratio less than the known concentration ratio in the sample indicates that the particles are too large to be completely atomized and ionized in the plasma, leading to low results for Th (since Th is not as readily atomized and ionized in the plasma). This study was conducted using NIST reference glass standards; the Th/U concentration ratios for these samples are near unity (see Tables V and VI). Measurements were taken by both scanning the laser over the sample surface and sampling at a single spot. Results of the fs-LA-ICP-MIC-MS analyses during the site test were compared to similar analyses of the reference standards performed with a) a commercial $n s$-LA system connected to a sequential-scanning ICP-MS at Ames Laboratory and with b) the same fsLA system connected to the sequential-scanning ICP-MS. The results are shown in Table V for scanning LA sampling and in Table VI for single-spot LA sampling.

It should be noted that the reported ${ }^{232} \mathrm{Th} /{ }^{238} \mathrm{U}$ values for the sequential-scanning ICP-MS were obtained after the instrument was optimized with a $100 \mathrm{ppb} \mathrm{U}$ tuning solution for maximum signal intensity. The results are presented to illustrate the improvement in accuracy and 
precision obtained with the state-of-the-art fs-LA-ICP-MIC-MS system. Experiments in our lab have shown that varying the scanning ICP-MS operating parameters, such as gas sample flow, can readily affect the measured ${ }^{232} \mathrm{Th} /{ }^{238} \mathrm{U}$ values. Adjusting various plasma and MS parameters affects the mass bias observed in these measurements. These tests illustrate that independent ratios obtained at optimized signal levels are much closer to the certified values using fs-LA compared to ns-LA. They are close enough that accurate inter-elemental ratios may be obtained with little optimization or with non-matrix-matched standards. We still need to perform tests to determine whether any residual mass bias observed using fs-LA could be corrected using liquid standards for normalization, something that could be not done previously with ns-LA sampling.

Since $f s$-LA greatly minimizes fractionation during sampling, measurements taken with the fs-LA-ICP-MIC-MS system yield ${ }^{232} \mathrm{Th} /{ }^{238} \mathrm{U}$ values much closer to the certified stoichiometric ratios for these samples. This is readily illustrated in Tables V and VI. The precision obtained for both single-spot LA and laser scanning analyses are significantly improved with the state-of-the-art system compared to the commercial ns-LA and scanning ICPMS system, as shown in Tables V and VI.

\section{Conclusion}

This study demonstrated significant improvement in the precision and accuracy in the measurement of uranium oxides using a state-of-the-art $f s$-LA-ICP-MIC-MS system compared to a conventional commercial ns-LA-ICP-MS system. A six-fold increase in precision was observed during the site test due to the incorporation of $f$ s-laser sampling and the multi-ion collector MS, meeting one of this project's most significant goals. There is excellent agreement between the measured 100-shot ablation ${ }^{235} \mathrm{U} /{ }^{238} \mathrm{U}$ values and the certified values. With the 
exception of the analysis of one depleted uranium oxide sample, the differences between the measured values and the certified values were less than $0.5 \%$.

Thus single-shot LA-ICP-MS can readily differentiate between very small samples of depleted, natural, and enriched uranium samples. From the reported data, a known natural abundance sample particle would yield a measurement within a 0.723 to $0.728 \%$ confidence range $95 \%$ of the time. Similarly, a sample that resulted in a measured value of $0.725 \%{ }^{235} \mathrm{U}$ would be $95 \%$ likely to lie in the prediction range of 0.707 to $0.742 \%$, based on the calibration and standards used during the test at ORNL.

This study also demonstrated that $f s$-LA greatly minimizes fractionation during sampling, thereby improving inter-elemental ratio analyses of samples. Measurements taken with the fsLA-ICP-MIC-MS system yielded ${ }^{232} \mathrm{Th} /{ }^{238} \mathrm{U}$ values much closer to the certified stoichiometric ratios for these samples, compared to the results obtained previously for ns-LA-ICP-MS.

\section{References}

1. Liu, C.; Mao, X. L.; Mao, S. S.; Zeng, X.; Greif, R.; Russo, R. E. “Comparison of ultraviolet femtosecond and nanosecond laser ablation ICP-MS Analysis on glass, monazite, and zircon” Anal. Chem. 2003, 75, 6184-6190; “Nanosecond and femtosecond LA of brass: particulate and ICPMS measurements” Anal Chem. 2004, 76, 379-383.

2. Wang, Z.; Hattendorf, B.; Günther, D. "Vaporization and ionization of laser ablation generated aerosols in an inductively coupled plasma mass spectrometer - implications from ion distribution maps” J. Anal. At. Spectrom., 2006, 21, 1143-1151.

3. Pisonero, J.; Günther, D. "Femtosecond laser ablation inductively coupled plasma mass spectrometry: Fundamentals and capabilities for depth profiling analysis” Mass Spectrom. 
Reviews, 2008, 27, 609-623; Koch, J.; Günther, D. “Femtosecond laser ablation inductively coupled plasma mass spectrometry: achievements and remaining problems” Anal. Bioanal. Chem., 2007, 387, 149-153.

4. Joshua Messerly, Nathan Saetveit, Stan Bajic, David Baldwin, and Sam Houk, “Analysis Of Enriched and Depleted Uranium Oxide Powders by LA-ICP-MS - Final Report,” Ames Laboratory Report IS-5174 (2005). 
Table I. Multi-ion collector array configuration used during the ORNL test. IC\# = designation of ion counter.

\begin{tabular}{|l|l|l|l|l|l|l|l|}
\hline IC2 & IC3 & IC4 & IC5 & IC6 & IC7 & L4 & IC8 \\
\hline${ }^{231} \mathrm{~Pa}$ & ${ }^{232} \mathrm{Th}$ & ${ }^{233} \mathrm{U}$ & ${ }^{234} \mathrm{U}$ & ${ }^{235} \mathrm{U}$ & ${ }^{236} \mathrm{U}$ & $\mathrm{m} / \mathrm{z} 237$ & ${ }^{238} \mathrm{U}$ \\
\hline
\end{tabular}

Table II. Faraday cup configuration used during the ORNL test. L\# or H\# = designation of Faraday cup detector.

\begin{tabular}{|l|c|c|c|c|c|c|c|}
\hline & L1 & L2 & L3 & Center & H1 & H2 & H3 \\
\hline $\begin{array}{l}\text { Configuration for } \\
\begin{array}{l}\text { Uranium Oxide } \\
\text { Measurements }\end{array}\end{array}$ & ${ }^{234} \mathrm{U}$ & ${ }^{235} \mathrm{U}$ & ${ }^{236} \mathrm{U}$ & $\mathrm{m} / \mathrm{z} 237$ & ${ }^{238} \mathrm{U}$ & ${ }^{239} \mathrm{Pu}$ & ${ }^{----}$ \\
\hline $\begin{array}{l}\text { Configuration for } \\
\text { Measurements } \\
\text { Measi } \mathrm{U}\end{array}$ & ${ }^{232} \mathrm{Th}$ & ${ }^{233} \mathrm{U}$ & ${ }^{234} \mathrm{U}$ & ${ }^{235} \mathrm{U}$ & ${ }^{236} \mathrm{U}$ & $\mathrm{m} / \mathrm{z} 237$ & ${ }^{238} \mathrm{U}$ \\
\hline
\end{tabular}

Table III. Measured ${ }^{235 / 238}$ U for Uranium Oxide in Collodion Analyzed by fs -LA-ICP-MIC-MS.

\begin{tabular}{|c|c|c|c|c|c|c|c|c|}
\hline & \multicolumn{4}{|c|}{ Day 1} & \multicolumn{4}{|c|}{ Day 2} \\
\hline$\left({ }^{235 / 238} \mathrm{U}\right.$ atom ratio $)$ & Avg & $\%$ RSD & reps & \% Dif & Avg & $\%$ RSD & reps & \% Dif \\
\hline 0.0528 & 0.052906 & \begin{tabular}{|l|}
0.33 \\
\end{tabular} & 4 & 0.20 & 0.052983 & 0.23 & 7 & 0.35 \\
\hline 0.0314 & 0.031396 & 0.41 & 4 & -0.01 & 0.031433 & 0.35 & 7 & 0.11 \\
\hline 0.0156 & 0.015669 & 0.73 & 5 & 0.44 & 0.015556 & 0.52 & 6 & -0.28 \\
\hline 0.00726 & 0.007289 & 0.43 & 6 & 0.40 & 0.007253 & 0.34 & 5 & -0.10 \\
\hline 0.00509 & 0.005101 & 0.54 & 4 & 0.21 & 0.005071 & 0.41 & 5 & -0.38 \\
\hline 0.000176 & 0.000174 & 0.52 & 5 & -1.17 & 0.000175 & 0.65 & 6 & -0.30 \\
\hline
\end{tabular}


Table IV. Uranium Oxide in Collodion measured on a Scanning ICP-MS.

\begin{tabular}{|c|c|c|c|c|c|c|c|c|}
\hline & \multicolumn{4}{|c|}{$n s$-LA, scanning MS } & \multicolumn{4}{|c|}{ fs -LA, scanning MS } \\
\hline$\left({ }^{235 / 238} \mathrm{U}\right.$ atom ratio $)$ & Avg & $\%$ RSD & reps & $\%$ Dif & Avg & $\%$ RSD & reps & $\%$ Dif \\
\hline \begin{tabular}{|c|}
0.0528 \\
\end{tabular} & 0.04878 & 7.25 & 5 & -7.61 & 0.04676 & 27.93 & 6 & -11.44 \\
\hline 0.0314 & 0.03359 & 19.87 & 5 & 6.97 & 0.03035 & 7.29 & 6 & -3.33 \\
\hline 0.0156 & 0.01862 & 33.25 & 5 & 19.38 & 0.01545 & 8.08 & 6 & -0.96 \\
\hline 0.00726 & 0.00502 & 50.18 & 5 & -30.91 & 0.00652 & 17.47 & 6 & -10.19 \\
\hline 0.00509 & 0.00414 & 23.67 & 5 & -18.57 & 0.00491 & 1.06 & 6 & -3.54 \\
\hline 0.000176 & 0.00017 & 23.07 & 5 & -4.91 & 0.00018 & 28.55 & 6 & 1.08 \\
\hline
\end{tabular}

Table V. Measured Th/U Ratios obtained for Scanning Laser Ablation on NIST Glass Standards.

\begin{tabular}{|c|c|c|c|c|c|c|c|c|c|}
\hline LA, MS system & \multicolumn{3}{|c|}{610} & \multicolumn{3}{c|}{612} & \multicolumn{3}{c|}{614} \\
\hline \multirow{3}{*}{$n s$-LA, scanning MS } & Avg & $\%$ RSD & $\%$ Diff & Avg & $\%$ RSD & $\%$ Diff & Avg & \%SD & $\%$ Diff \\
\cline { 2 - 11 }$y$ & 0.52 & 7.28 & -49.11 & 0.51 & 9.98 & -50.86 & 0.42 & 2.08 & -54.79 \\
\hline fs -LA, scanning MS & 0.86 & 2.20 & -15.74 & 0.91 & 0.96 & -12.91 & 0.80 & 2.94 & -14.07 \\
\hline fs -LA, multicollector MS & 0.96 & 0.83 & -6.30 & 0.96 & 1.59 & -7.38 & 0.86 & 0.93 & -7.59 \\
\hline Certified Value $\left.{ }^{232} \mathrm{Th} /{ }^{238} \mathrm{U}\right)$ & 1.02 & & & 1.04 & & & 0.94 & & \\
\hline
\end{tabular}

Table VI. Measured Th/U Ratios obtained for Single-Spot Laser Ablation on NIST Glass Standards.

\begin{tabular}{|c|c|c|c|c|c|c|c|c|c|}
\hline LA, MS system & \multicolumn{3}{|c|}{610} & \multicolumn{3}{c|}{612} & \multicolumn{3}{c|}{614} \\
\hline \multirow{2}{*}{$n s$-LA, scanning MS } & Avg & $\%$ RSD & $\%$ Diff & Avg & $\%$ RSD & $\%$ Diff & Avg & $\%$ RSD & \% Diff \\
\cline { 2 - 11 }$y$ & 0.64 & 15.28 & -36.80 & 0.53 & 30.72 & -49.41 & 0.52 & 1.75 & -44.38 \\
\hline$f s$-LA, scanning MS & 1.02 & 10.72 & 0.18 & 0.97 & 18.87 & -6.69 & 0.94 & 16.82 & 1.02 \\
\hline fs -LA, multicollector MS & 0.99 & 1.16 & -2.84 & 0.99 & 1.87 & -4.90 & 0.91 & 4.03 & -2.46 \\
\hline Certified Value $\left({ }^{232} \mathrm{Th} /{ }^{238} \mathrm{U}\right)$ & 1.02 & & & 1.04 & & & 0.94 & & \\
\hline
\end{tabular}




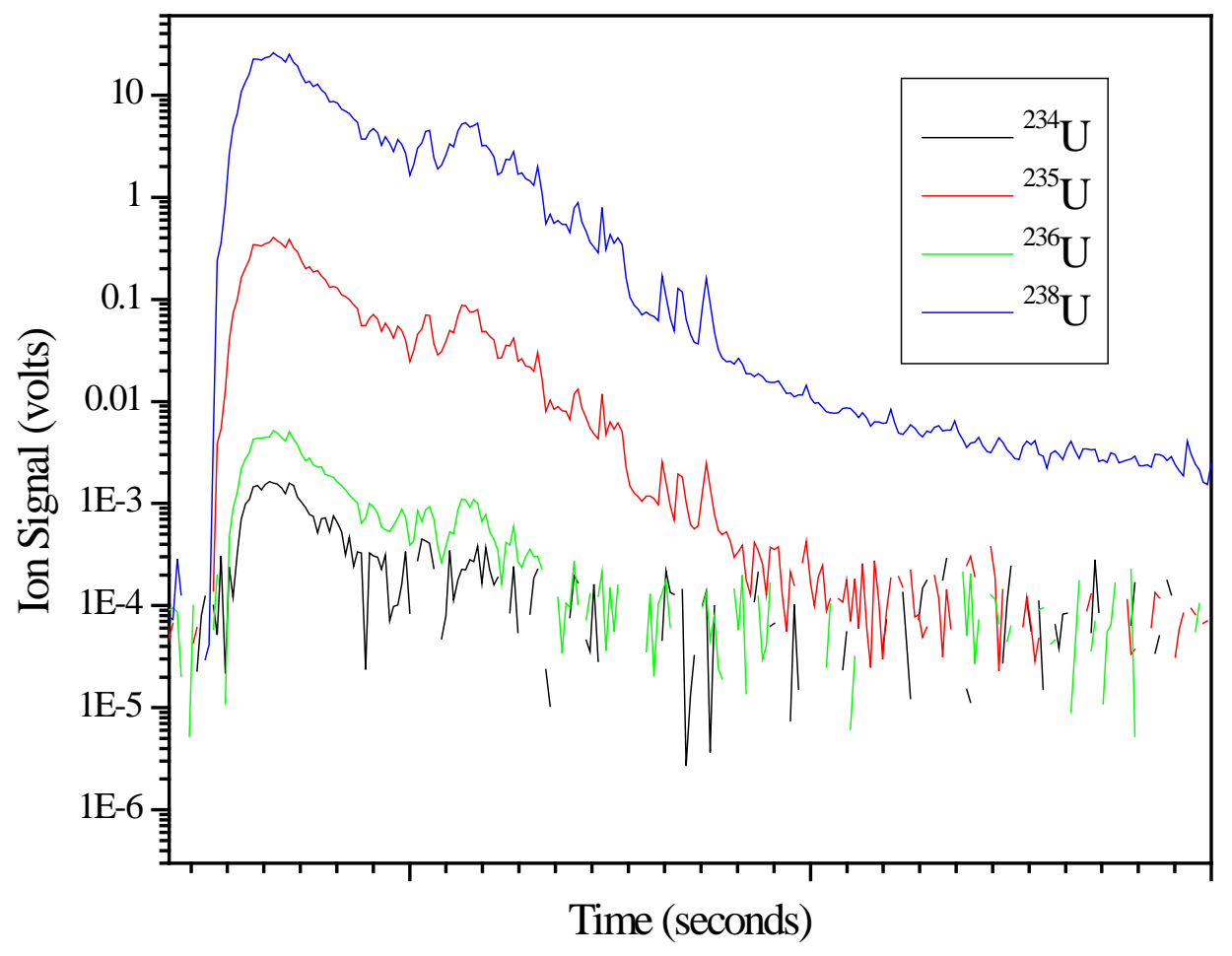

Figure 1. Typical transient signals from a single 100-shot fs-LA-ICP-MIC-MS analysis (Faraday cup data) of an enriched uranium oxide sample containing $1.5 \%{ }^{235} \mathrm{U}$. 


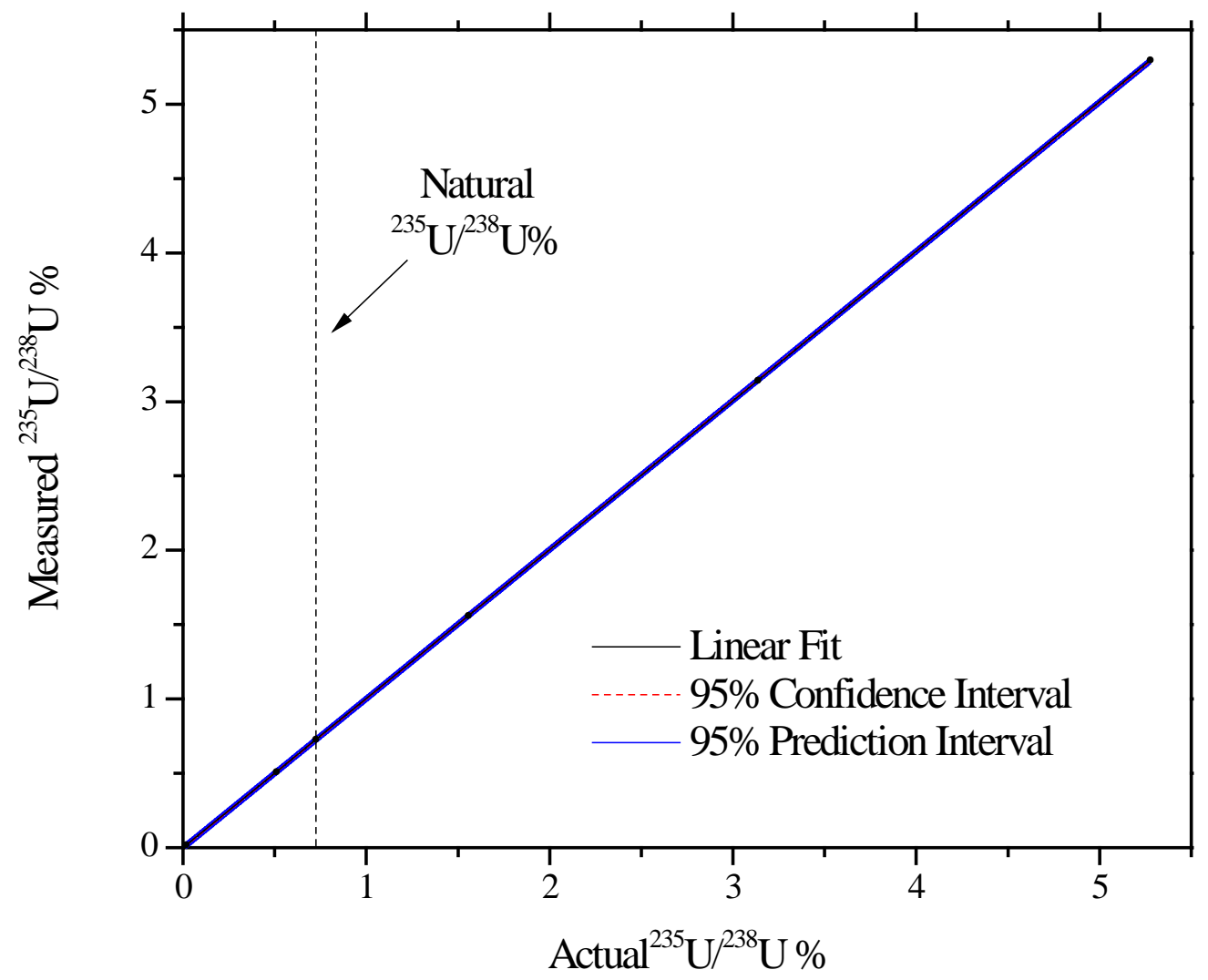

Figure 2. Linear-regression fit of the entire dataset of uranium oxide samples, analyzed by 100shot fs-LA-ICP-MIC-MS at ORNL (Faraday cup data). The 95\% upper and lower confidence intervals and the 95\% upper and lower prediction intervals are plotted. 


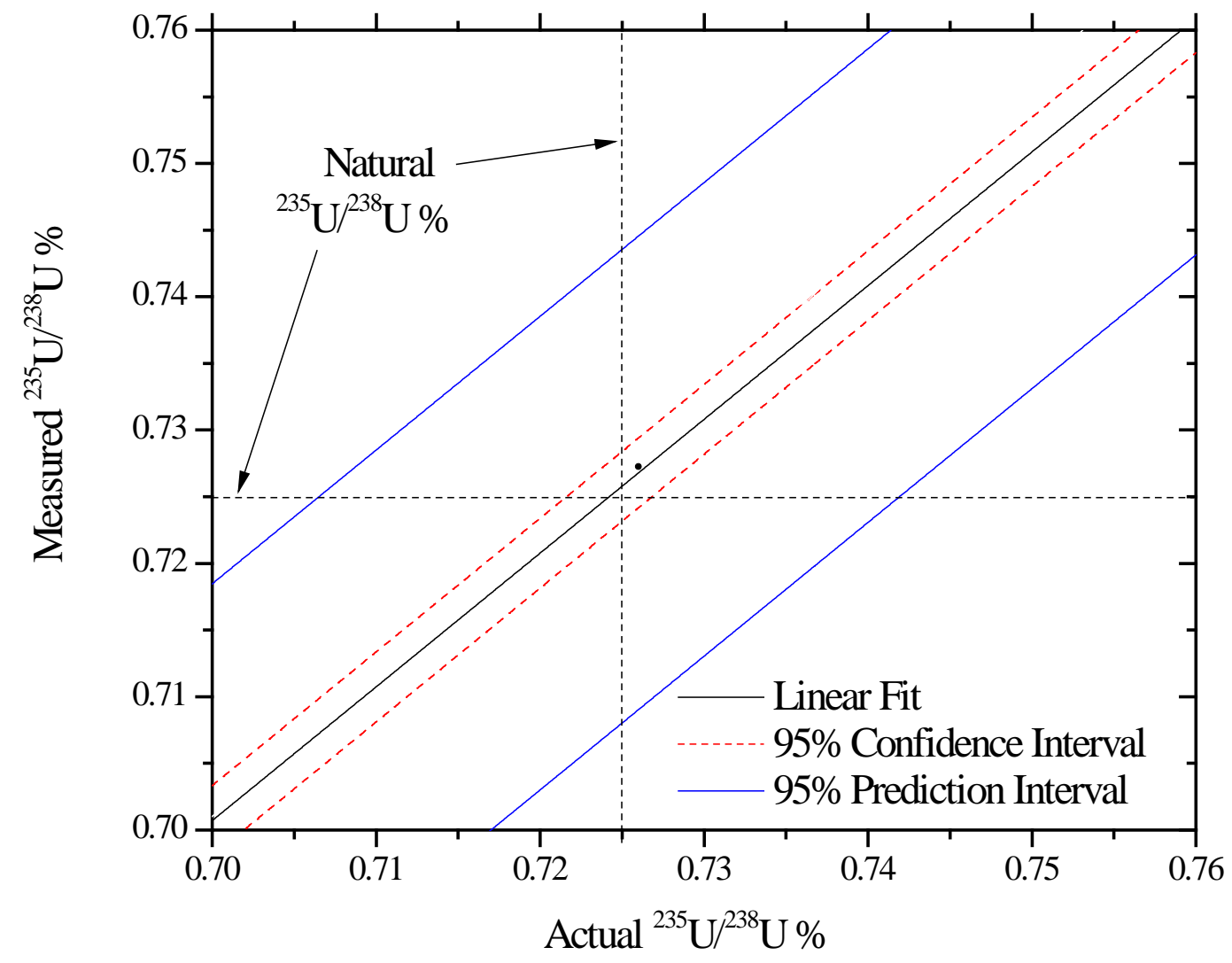

Figure 3. An expanded view (approximately centered around natural isotope abundance uranium) of the least-squares fit of the entire dataset acquired at ORNL. The 95\% upper and lower confidence intervals and the 95\% upper and lower prediction intervals are plotted. The dashed vertical and horizontal lines represent natural isotope abundance uranium ratio. 